\title{
Quantum Model for Ferromagnetic Thin Films with an Alternating Crystal Field
}

\author{
J. KECER \\ Department of Physics, Technical University \\ Park Komenského 2, 04001 Košice, Slovak Republic
}

AND S. TULEJA

High School Humenné, Komenského 2, 06001 Humenné, Slovak Republic

Within the framework of many-body Green's function theory there are studied the properties of the quantum Blume-Capel model for ferromagnetic films with an alternating single-ion anisotropy on the odd atomic layers and on the even ones. We analyse various possible phase diagrams for the surface exchange couplings and the single-ion anisotropy parameters.

PACS numbers: 75.10.Jm, 75.30.Ds, 75.30.Kz, 75.70.Ak

\section{Model and method}

The ferromagnetic Blume-Capel-Ising (BCI) model has been studied within the mean field approximation [1], the effective field theory [2], the two-spin cluster approximation in the cluster expansion method [3, 4], Monte Carlo simulations [5], a thermodynamically self-consistent theory based on an Ornstein-Zernike approximation [6], the exact solution based on the Bethe lattice by means of the exact recursion relations [7]. Most of the studies mentioned above displays also the existence of a tricritical point at which the phase transition changes from second order to first order when the value of $K_{2}$ becomes negative. Our work represents the first atempt to consider a quantum version of BCI model. Within quantum Blume-Capel (QBC) model we will study the influence of the enhancement of the surface exchange coupling and the alternative single-ion anisotropy $K_{2}(1)$ on the odd atomic layers and $K_{2}(2)$ on the even ones on the critical behaviour of thin ferromagnetic films.

The Hamiltonian of the considered system consists a Heisenberg exchange interaction with strength $J_{i j}>0$ between nearest neighbour lattice sites, an exchange anisotropy with strength $D>0$, and a second-order single-ion anisotropy with strength $K_{2}>0$ : 


$$
H=-\frac{1}{2} \sum_{\langle i, j\rangle} J_{i j}\left(S_{i}^{-} S_{j}^{+}+S_{i}^{z} S_{j}^{z}\right)-\frac{1}{2} D \sum_{\langle i, j\rangle} S_{i}^{z} S_{j}^{z}+K_{2} \sum_{i}\left(S_{i}^{z}\right)^{2} .
$$

Here the notation $S_{k}^{ \pm}=S_{k}^{x} \pm \mathrm{i} S_{k}^{y}(k=i, j)$ is introduced, where $i$ and $j$ are lattice site indices and $\langle i j\rangle$ indicates summation over nearest neighbour spins in the atomic layers and in the adjacent layers for sc lattice with (001) surfaces, $K_{2}$ takes the different values: $K_{2}(1)$ on the odd layers and $K_{2}(2)$ on the even ones and the exchange parameter takes the value $J_{S}$ at the surfaces and $J$ inside of the film.

In order to treat the problem for general spin $S$, we need the following Green functions $G_{i j}^{l}(\omega)=\left\langle\left\langle S_{i}^{+} ;\left(S_{j}^{z}\right)^{l} S_{j}^{-}\right\rangle\right\rangle_{\omega}$, where $l \geq 0$ is integer, necessary for dealing with higher spin values $S$. The equations of motion for calculation of $G_{i j}^{l}(\omega)$ are $\omega G_{i j}^{(l)}(\omega)=A_{i j}^{(l)} \delta_{i j}+\left\langle\left\langle\left[S_{i}^{+} ; H\right] ;\left(S_{j}^{z}\right)^{l} S_{j}^{-}\right\rangle\right\rangle_{\omega}$ with the inhomogeneities $A_{i j}^{(l)}=\left\langle\left[S_{i}^{+},\left(S_{j}^{z}\right)^{l} S_{j}^{-}\right]\right\rangle$, where $\langle\cdots\rangle=\operatorname{Tr}\left(\cdots \mathrm{e}^{-\beta H}\right) / \operatorname{Tre}^{-\beta H}$ denotes the thermodynamic expectation value, the brackets $[\cdots]$ denote the commutator, $\beta=1 / k_{\mathrm{B}} T$ and $\delta_{i j}$ is the Kronecker symbol. The higher Green functions due to the exchange interaction term are decoupled by Tyablikov-Bogolyubov (or RPA) approximation [8]. For the terms stemming from the single-ion anisotropy we have chosen the Anderson-Callen decoupling procedure [9] gives good results [10, 11] for the magnetization if the anisotropy parameter $K_{2}$ is much smaller than the parameter of the exchange coupling. Using the eigenvector method (EVM) described, for example, in [12-14] we obtain after a two-dimensional Fourier transform to momentum space, the $L$ coupled equations of motion for Green's functions $G_{\nu \mu}^{(l)}(\boldsymbol{q}, \omega)$ of layer labeled by $\mu$. By using the spectral theorem, for the spontaneous magnetization per site in each atomic layer of the film with spin $S$ we obtain

$$
\left\langle S_{\mu}^{z}\right\rangle_{S}=\frac{\left(S-\Phi_{\mu \mu}\right)\left(1+\Phi_{\mu \mu}\right)^{2 S+1}+\left(1+S+\Phi_{\mu \mu}\right) \Phi_{F \mu \mu}^{2 S+1}}{\left(1+\Phi_{\mu \mu}\right)^{2 S+1}-\Phi_{\mu \mu}^{2 S+1}}
$$

where $\Phi_{\mu \mu}=\frac{1}{\pi^{2}} \int_{0}^{\pi} \int_{0}^{\pi} \mathrm{d} q_{x} \mathrm{~d} q_{y} \sum_{\nu=1}^{L} \sum_{\kappa=1}^{L} R_{\mu \nu} \mathcal{E}_{\nu \kappa} \delta_{\nu \kappa} L_{\kappa \mu} . \quad \boldsymbol{R}$ is matrix whose columns are the right eigenvectors of matrix $\boldsymbol{P}_{L}$ of $L$ coupled equations of motion, its inverse $\boldsymbol{L}=\boldsymbol{R}^{-1}$ contains the left eigenvectors as rows, $\mathcal{E}_{\nu \kappa} \delta_{\nu \kappa}=\left(\mathrm{e}^{\beta \omega_{\nu}}-1\right)^{-1}$ are matrix elements of a diagonal matrix $L \times L$ and $\omega_{\nu}$ are the eigevalues of matrix $\boldsymbol{P}_{L}$. The reduction Curie temperature $k_{\mathrm{B}} T_{\mathrm{C}}^{f} / J$ of the film we get, for example for spin $S=1$, from the set of $L$ equations: $k_{\mathrm{B}} T_{\mathrm{C}}^{f} / J=2 / 3 \widetilde{\Phi_{2}}$, $\left\langle S_{2}^{z}\right\rangle=\widetilde{\Phi}_{1} / \widetilde{\Phi}_{2}, \cdots,\left\langle S_{L}^{z}\right\rangle=\widetilde{\Phi}_{1} / \widetilde{\Phi}_{L}$ where the overtilde designates a scaled quantity in terms of $\left\langle S_{1}^{z}\right\rangle$.

\section{Results}

First we consider in Fig. 1 the phase diagrams in $\left(K_{2}(1) / J, k_{\mathrm{B}} T_{\mathrm{C}}^{f} / J\right)$ plane when $K_{2}(2) / J=0.1$ (Fig. 1A) and in $\left(K_{2}(2) / J, k_{\mathrm{B}} T_{\mathrm{C}}^{f} / J\right)$ plane when $K_{2}(1) / J=0.01$ (Fig. 1B). In both cases we observe cross-over points at which Curie temperature of thin film does not depend on film thickness: $K_{2}^{\mathrm{C}}(1) / J$ and $K_{2}^{\mathrm{C}}(2) / J$. The tricritical points (denoted by $C$ ) are marked by filled circles. 


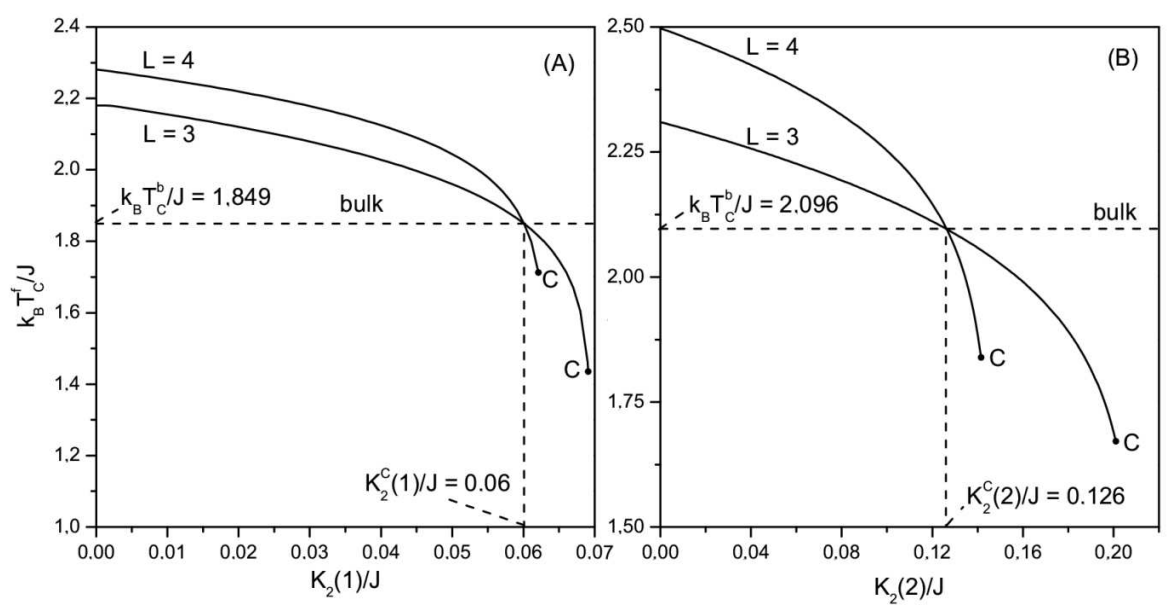

Fig. 1. Film Curie temperature $k_{\mathrm{B}} T_{\mathrm{C}}^{f} / J$ as a function: (A) of the anisotropy parameter $K_{2}(1) / J$ when $K_{2}(2) / J=0.1$; (B) of anisotropy parameter $K_{2}(2) / J$ when $K_{2}(1) / J=$ 0.01 for films with with spin $S=1$, with thicknesses $L=3$ and 4 in the case when $\Delta_{S}=J_{S} / J=1, D / J=0.01$. The dashed line labeled by "bulk" corresponds to the bulk Curie temperature.
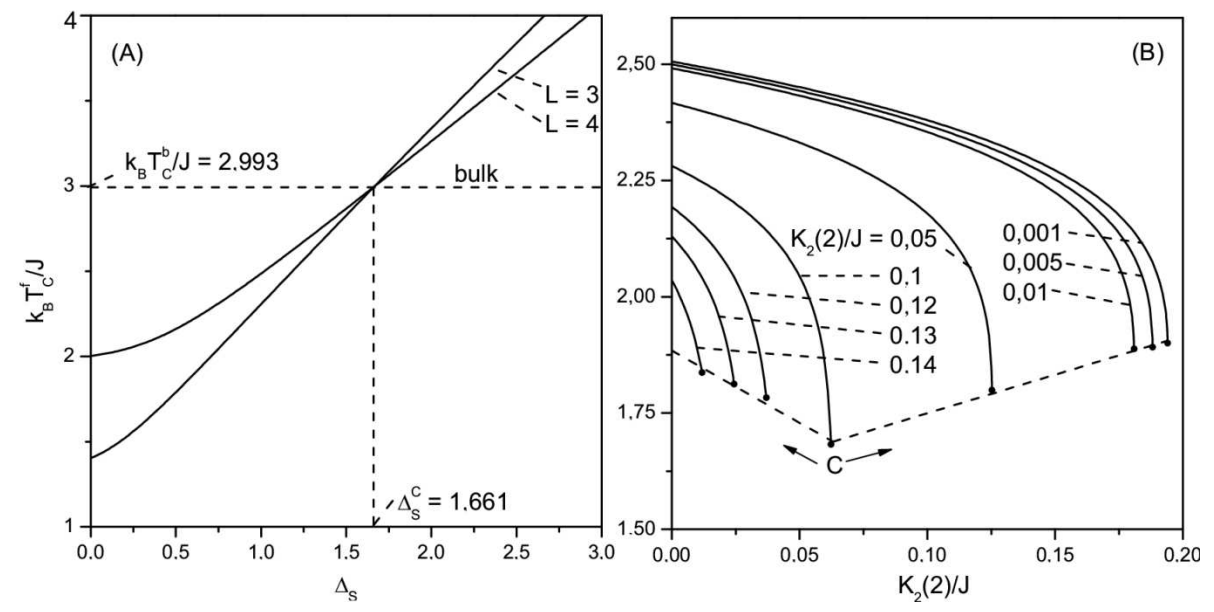

Fig. 2. Film Curie temperature $k_{\mathrm{B}} T_{\mathrm{C}}^{f} / J$ : (A) as a function of parameter $\Delta_{S}$ for films with spin $S=1$, with thicknesses $L=3$ and 4 when $D / J=0.01, K_{2}(1) / J=0.005$, $K_{2}(2) / J=0.01$; (B) as a function of anisotropy parameter $K_{2}(1) / J$ for different $K_{2}(2) / J$ when $S=1$ for film with thickness $L=4, \Delta_{S}=14, D / J=0.01$.

In Fig. $2 \mathrm{~A}$ there is plotted the phase diagram in $\left(\Delta_{S}, k_{\mathrm{B}} T_{\mathrm{C}}^{f} / J\right)$ plane. The critical parameter $\Delta_{S}^{\mathrm{C}}=J_{S}^{\mathrm{C}} / J$ represents other cross-over point. The cross-over points can be observed only in the cases: anisotropic exchange couplings when the surface exchange coupling differs from the bulk one; when the single-ion anisotropy is dif- 
ferent in the odd and in the even atomic layers of the films, etc. In Fig. 2B there are plotted the tricritical points in the phase diagrams in the $\left(K_{2}(1), k_{\mathrm{B}} T_{\mathrm{C}}^{f} / J\right)$ plane for different values of the single-ion anisotropy parameters $K_{2}(2) / J$.

\section{Acknowledgments}

Financial support of this work was provided by grants $1 / 2008 / 05$ and 1/4013/07 of Grant Agency for Science, Slovak Republic.

\section{References}

[1] J.A. Plascak, J. Moreira, F.C. Sá Barreto, Phys. Lett. A 173, 360 (1993).

[2] L. Peliti, M. Saber, Phys. Status Solidi B 195, 537 (1996).

[3] V. Ilkovič, Phys. Status Solidi B 192, K7 (1995).

[4] V. Ilkovič, Physica A 234, 545 (1996).

[5] D. Pena Lara, J.A. Plascak, Int. J. Mod. Phys. B 12, 2045 (1998).

[6] S. Grolau, Phys. Rev. E 65, 056130 (2002).

[7] O. Özsoy, E. Albayrak, M. Keskin, Physica A 304, 443 (2002).

[8] W. Gasser, E. Heiner, K. Elk, Greensche Funktionen in Festkörper- und Vielteilchenphysik, Wiley-VCH Verlag, Berlin 2001.

[9] H.B. Callen, Phys. Rev. 130, 890 (1963).

[10] P. Fröbrich, P.J. Jensen, P.J. Kuntz, Eur. Phys. J. B 13, 477 (2000).

[11] V. Ilkovič, Phys. Status Solidi B 240, 213 (2003).

[12] V. Ilkovič, Acta Physica Universitatis Comenianae XLIII, 53 (2002).

[13] V. Ilkovič, Acta Physica Universitatis Comenianae XLIV-XLV, 3 (2003).

[14] J. Kecer, V. Ilkovič, Central European J. Phys. 4, 461 (2006). 